\title{
Online focus groups and qualitative research in the social sciences: their merits and limitations in a study of housing and youth
}

\author{
Tom Moore* \\ University of Sheffield \\ Kim McKee \\ University of St Andrews \\ Pauline McLoughlin \\ RMIT University
}

\begin{abstract}
This paper considers the use of online focus groups as a method for conducting qualitative research in the social sciences. Researchers have increasingly utilised online focus groups involving live, synchronous chat room interactions. However, to date there has been little insight and a lack of discussion as to the applicability of online focus groups in the social sciences. Reflecting on a study of young people's housing opportunities and financial welfare in the UK, this paper considers the advantages and limitations of online qualitative methods. We argue that online methods offer significant advantages, especially in longer-term studies crossing time and space, but that their design and implementation raise methodological challenges, with implications for the depth and insight of the knowledge produced. Their use for social science research therefore requires reflexivity and adjustment, including attention to the positionality of the researcher, the nature and level of participant involvement, and adjustment to the loss of non-verbal cues and interactions found in conventional qualitative research. This paper advances knowledge on the opportunities of and challenges to online methodologies, and highlights how creative use of webbased technology can support social scientists conducting qualitative research.
\end{abstract}

Keywords: online research; focus groups; qualitative research; housing research; internet; young people. 
p. 18. Online focus groups and qualitative research in the social sciences: their merits and limitations in a study of housing and youth

\section{Introduction}

Focus groups are an established method of collecting research data in the social sciences, bringing together people with mutual characteristics or interests to offer individual and collective insights into particular topics (Morgan, 1996: 130). Online focus groups capitalise on the increasing use of the internet as a communication tool. They currently take two forms: synchronous, involving real-time live chat comparable to conversational interactions of face-to-face focus groups (Fox et al., 2007), or asynchronous, using 'static' text-based communication such as forums and email lists (Gaiser, 1997; Kenny, 2005). Yet, while the use of online focus groups has been discussed in other social sciences such as nursing and health research (Kenny, 2005; Fox et al., 2007; Williams et al., 2012; O'Connor et al., 2014), there has been a lack of discussion as to how they might be applied in disciplines such as social policy, geography, urban studies, and related areas. Based on a study of young people's housing opportunities and welfare in the UK, this paper reflects on the use of synchronous online focus groups. We consider their advantages and limitations, highlighting the methodological implications that suggest a need for significant reflexivity by researchers that acknowledges the influence that different interactions (in this case through web-based, non-verbal platforms) have on participant relationships and the production of knowledge (Bourdieu, 1992).

\section{The collective conversations and potential of online focus groups}

Focus groups share similar epistemological concerns to other qualitative methods, as they are concerned with the depth and meaning of participants' experiences, but are differentiated on the basis of certain features. Advocates argue that focus groups are reflexive and empowering experiences for participants, as researchers encourage participants to share views and experiences through group conversations (Goss and Leinbach, 1996). These "collaborative research performances" (Bosco and Herman, 2010) are thought to better reflect the socially constructed nature of knowledge, as participants are encouraged to query, question and explain their viewpoints through group interaction, teasing out complementary and argumentative interpretations of the research topic (Goss and Leinbach, 1996; Kamberelis and Dimitraidis, 2013). Bagnoli and Clark (2010: 104) contend that this changes the way that research data is produced, as "it is in the interaction between participants, rather than between participant and researcher, that generates data." This gives more space for participants to discuss issues they deem significant, emphasising the role of group dynamics in shaping the knowledge that is produced. Non-verbal cues such as body language, visual signs of agreement or disagreement, and physical identities can also affect the flow and direction of focus groups, allowing the researcher to observe how social contexts may influence what is said, and when, in the group setting.

However, the potential of focus groups depends upon overcoming a range of methodological limitations associated with their use, including the size and composition of groups, practical constraints of location and timing, and the positionalities (including class, gender, ethnicity, and lived experiences) of the researcher and participants (Holbrook and Jackson, 1996; Hollander, 2004; Hopkins, 2007). The influence of cultural norms and expectations is an important consideration, as people may conform to dominant ideas and beliefs established by social, geographical and political contexts (Koch, 2013).

While use of online focus groups has grown, the majority of published discussions focus on asynchronous groups (Gaiser, 1997; Mann and Stewart, 2000; Kenny, 2005; 
p. 19. Online focus groups and qualitative research in the social sciences: their merits and limitations in a study of housing and youth

Williams et al., 2012). Themes from these discussions include: the potential for online groups to bring together geographically distant individuals and groups in web-based settings; practical advantages of avoiding costly and difficult transcription; and the ability to facilitate greater participation and disclosure for users who are comfortable with the social uses, and privacy, of the internet. However, there has been little reflection in the social sciences on the use of online focus groups, particularly regarding how they impinge upon group dynamics and collaborations of participants, and how moderators and participants adapt to online environments, where human and non-verbal interaction may be diminished. The remainder of this paper contributes to our understanding of the advantages and limitations of online synchronous focus groups. Reflecting on their application in a qualitative study of housing and youth in the UK, we highlight a range of issues including the effectiveness of online recruitment, the size and composition of groups, and the role of the researcher in facilitating discussion.

\section{Background to our study of housing and youth}

From 2013-14, we used online focus groups to research the housing and financial welfare of young adults aged 18-35 in the UK. Young people face many difficulties with housing, including the cost of housing, difficulties in raising deposits and securing mortgages for ownership, and problems with accessing and costs of rental housing (Moore, 2013). These difficulties are exacerbated by changes to welfare support systems, and are predicted to have long-term implications for the future welfare of young people (McKee, 2012). Ten case study areas were selected in rural and urban areas of Northern Ireland (Belfast and Ballymena), Wales (Gwynedd and Merthyr), England (Cornwall, Surrey and Sheffield) and Scotland (North Lanarkshire, the Scottish Borders, and Edinburgh). We aimed to conduct 20 online focus groups, though as Table 1 shows only 10 were eventually conducted.

We chose synchronous online focus groups as a method to research the implications of the issues facing young people. As we were conducting research across the UK, this choice was made to overcome traditional time and spatial constraints of qualitative research, as well as to engage young people familiar with the social uses and conventions of online communication. Recent statistics show that 87 per cent of adults aged 16-24 in the UK use social networking as a form of internet communication (Office for National Statistics, 2013). The same report shows that social networking has also replaced email as the most popular internet activity. By facilitating active, participant-led discussions, real-time online focus groups also have the advantage of producing some of the same forms of collaborative knowledge as conventional face-to-face focus groups.

Table 1 summarises the 10 synchronous online focus groups in our study. These were held in six of the 10 case study areas across the UK and were conducted in real time through text-based chat rooms, hosted securely on a private server by a specialist provider of online qualitative research platforms. 
p. 20. Online focus groups and qualitative research in the social sciences: their merits and limitations in a study of housing and youth

Table 1: Number of focus groups conducted

\begin{tabular}{lll}
\hline Date of focus group & Case study area & $\begin{array}{l}\text { Number of } \\
\text { participants }\end{array}$ \\
\hline 16 April 2013 & Edinburgh & 3 \\
29 May 2013 & North Lanarkshire & 4 \\
3 July 2013 & Cornwall & 4 \\
14 November 2013 & Sheffield & 4 \\
21 November 2013 & Sheffield & 4 \\
10 December 2013 & Edinburgh & 4 \\
17 December 2013 & Scottish Borders & 3 \\
8 April 2014 & Surrey & 4 \\
\hline
\end{tabular}

In addition, 30 telephone interviews were conducted. This option was offered to ensure that digitally-excluded young people had an opportunity to participate in the study. This proved to be an important element of the research, as recruitment challenges meant that ten rather than 20 focus groups were held. Participation for both focus groups and interviews was incentivised through a small gift voucher reward, as an appreciation of participants' time.

All research was carried out with ethical approval and informed consent was gained from all participants. However, online qualitative research raises some distinct ethical questions for researchers. Firstly, the issue of privacy may be problematic, due to the unclear status of different sources of online information which may be used for research purposes. The British Psychological Society (2013) note that the distinction between public and private spaces is often blurred in online settings, as it is not always explicit which online spaces are perceived as 'private' or 'public'. Furthermore, the accessibility and permanence of online activities presents challenges distinct from face-to-face research contexts, as there is no scope for participants to opt out of providing information in a setting which is more public than a private encounter. This raises questions of anonymity and confidentiality. Secondly, there may be difficulties in obtaining valid consent, as research conducted online may make it difficult to verify certain relevant participant characteristics (such as age, gender, and ethnicity), while establishing the participants have properly engaged with valid consent procedures may also be difficult.

All participants were informed as to how the online data they provided would be electronically stored and used via a detailed information sheet. A password-protected portal provided by a respected provider was used to host the focus groups. The secure nature of this prohibited the possibilities for breach of confidentiality through, for example, the possible searching of published quotes using search engines. After a designated period of time, transcripts were removed from the online portal, further reducing potential breaches of confidentiality. The full name of participants was also not visible to anyone using the portal, with personal details stored offline. All participants were asked to complete a consent form, including clear statements that they were able to withdraw from participation in the study at any time. Finally, all participants were asked to complete a demographic questionnaire before taking part. This information was stored privately. While there remains a risk of false reporting, asking participants to complete this helped to reassure the researchers - as far as feasibly possible - that participants met the particular demographic characteristics required for the study.

We found the process of obtaining consent for online qualitative research similar to that of face-to-face contexts. However, the relative novelty of online research, coupled with the blurred distinction between public and private dialogues in online settings, 
p. 21. Online focus groups and qualitative research in the social sciences: their merits and limitations in a study of housing and youth

places a greater responsibility on researchers to protect the privacy and confidentiality of participants, as well as to ensure that the storage and use of information provided online is both secure for, and clear to, participants.

\section{Online focus groups in practice}

\section{Recruitment strategies}

Given that online focus groups utilise the internet, web-based settings are usually the most common methods of recruitment (Gaiser, 1997; O'Connor et al., 2014). Pertinently for our study, societal trends suggested a high level of use of online social networks such as Facebook and Twitter by our target sampling population (Office for National Statistics, 2013), allowing us to identify online networks as potentially productive for participant recruitment. Relevant Facebook pages were identified, such as youth services and those that promote local events and opportunities, and links to our project website were posted, with a short description of the study. While this proved to be productive, we were faced with some challenges. Gaiser (1997) notes the danger of intrusion in online communities and the risk of 'spamming' (intrusive and unsolicited messages and advertisements). Discretion was used by the researchers, firstly by ensuring the page used to promote the study - and therefore the interests of the social network community in question - was aligned with the research topic. Secondly, we requested permission from the page owner to post recruitment notices.

Using the social networking site Twitter proved to be the most fruitful recruitment technique. Twitter is an interactive micro-blogging website used by people and organisations to send succinct messages to each other in order to share online material, links, and images. Each Twitter account has a set of 'followers' who have chosen to subscribe to the messages sent by that account. Each user can share information with their followers, not only by posting a message but by 'retweeting' the messages of other users, thereby sharing information with a wider audience. The present popularity of Twitter has enabled its use by researchers as a method of online participant recruitment (O'Connor et al., 2014).

We asked organisations relevant to both the research topic and specific case study areas to tweet and retweet links to our project website and recruitment notice, including voluntary groups, housing providers, local newspapers, and youth support services. We understand these recruitment strategies as an online form of snowball sampling (O'Connor et al., 2014), as the sharing of information through retweeting travels through wider audiences and networks. Not only were a number of participants recruited through this method, but in some case study areas the process of retweeting helped to publicise the study to gatekeepers previously unknown to us, therefore extending the snowball sampling even further.

The web-based nature of the research also engaged people with disabilities or barriers that prevented them from taking part face-to-face, such as those with hearing difficulties. However, there were also limitations to online recruitment. Sharing information through popular social media accounts is an efficient way to recruit by reaching a large number of people, however this is based on assumptions that social media account users will match the target population of the research topic. For our study, there was uncertainty as to whether we were successfully targeting individuals with the required demographic traits of age and residence. While these criteria were quite broad, online recruitment methods may not be replicable or as successful for studies with narrower selection criteria. 
p. 22. Online focus groups and qualitative research in the social sciences: their merits and limitations in a study of housing and youth

While online recruitment can widen the pool of potential participants, its potential to act as a restrictive and exclusionary device should also be taken into account. There are still segments of the population that are excluded on the basis of socio-economic disadvantage, age, and geography. This was illustrated by the fact that seven of our ten focus groups were held in urbanised areas, rather than rural areas which traditionally face issues of digital exclusion (Townsend et al., 2013). Accordingly, we used offline mechanisms of sampling to complement the online components, using a conventional snowball sampling technique for recruitment. We engaged gatekeepers at relevant organisations in each case study area, including local housing associations, voluntary and community groups, local authorities, housing and financial support services, and organisations working with young people and families. These organisations communicated the study to their users on our behalf.

\section{The size, composition and hosting of online focus groups}

Decisions as to the size and composition of focus groups are linked to the purpose and sensitivity of the research topic. Smaller groups may provide opportunities to voice opinions, while larger groups may elicit a wider range of responses (Bedford and Burgess, 2001; Hopkins, 2007). We had initially aimed for focus group sizes of between four and eight participants. After a pilot focus group with eight colleagues, we adjusted group sizes to the lower end of this range, and in some cases conducted groups with three participants due to poor recruitment response rates or difficulties in identifying mutually convenient times for the group to take place. Our experience in the larger pilot group was also similar to the groups ran by Fox et al (2007), as the number and speed of overlapping discussion threads proved difficult to manage and follow.

The composition of focus groups is often based on key demographic traits that enhance familiarity and facilitate greater disclosure and discussion (Morgan, 1996: 143), although others advocate for heterogeneous groups on the basis that this encourages a diversity of viewpoints and avoids conformity with group consensus (Hollander, 2004). Decisions on the composition of our groups evolved throughout the study. Participants were recruited from a diverse range of social backgrounds and occupations, including students, graduates, single men living in supported accommodation, homeowners (sometimes with more than one property) and those with families. Given this diversity, efforts were made to create relatively homogenous groups as, for instance, the housing and financial challenges faced by 18 -year-old students living independently were likely to differ markedly from older participants who owned multiple properties.

The homogeneity of our groups allowed us to focus on aspects of housing and financial welfare affecting these individuals, enabling areas of commonality that stimulated discussion. On some occasions, it was necessary to have heterogeneous groups with varied demographics, according to levels of recruitment and availability. Whilst these still produced valuable insights, there was a risk of creating 'outsiders' who lacked areas of common ground with other participants. For instance, in one group of four participants, three were homeowners and one rented privately and did not intend to buy a home. Discussion tended to focus on aspects of homeownership, such as deposit-saving, costs of maintenance and the benefits or challenges of being a homeowner, some of which seemed to be beyond the interest or expertise of the other participant. This caused a dilemma for us in moderation, as we attempted to elicit contributions from the 'outsider' participant whilst trying to avoid disrupting the participant-led interactions and discussions of the other three participants. The lack of non-verbal cues or visual insight into the 'outsider's' mood exacerbated our concerns, thus exposing a disadvantage of online focus groups compared to the human interaction of their face-to-face equivalent. 
p. 23. Online focus groups and qualitative research in the social sciences: their merits and limitations in a study of housing and youth

While online focus groups have been advocated for their potential to overcome barriers to participation such as time and space, we found it challenging to arrange mutually convenient dates and times to suit our participants. Online groups usually occurred in the evenings to suit participants' availability. To develop rapport and maintain contact, the lead researcher contacted participants a number of times in the days preceding each group to organise participation. Despite this, some focus groups suffered from non-attendance. Reasons for this were sought from non-attendees and tended to relate to forgetfulness, which may be related to over-reliance on electronic communication rather than human interaction, as well as problems with computers or connecting to the internet. This suggests that online methods require additional faceto-face or telephone interactions to secure and maintain participation.

\section{Interactive procedures of data collection}

Our focus groups took place in text-based chat rooms, and involved a mixture of text-based questions and periodic use of a virtual whiteboard for brainstorming and idea sharing (discussed in the next section). Advocates of online communication in research have argued that virtual environments can enhance disclosure from participants, as anonymity can remove inhibitions and encourage people to contribute candid viewpoints without concerns as to the personal ramifications of their statements (Fox et al., 2007). Text-based interactions may also avoid impacts of dominant personalities or strong voices encountered in face-to-face environments (Hollander, 2004; Kenny, 2005). However, one of the major critiques of online research is that non-verbal cues are lost, and reliance on text-based exchanges creates focus groups that are "more task oriented and less interpersonal" (Fern, 2001: 68).

In our experience, interpersonal exchanges were not diminished by the virtual environment. This was helped by exercises used to establish rapport and encourage interaction. At the outset of each group, participants were asked to share their motivation for taking part. As groups were mostly segmented according to similar housing circumstances or tenures, participants often identified commonalities through shared motivations and circumstances. Encouraged by this connectedness, participants often conversed with each other directly rather than simply addressing the moderator

In turn, this rapport may also have harnessed disclosure. Participants were generally happy to share their anxieties in relation to personal issues of housing access and financial welfare, with insights offered into young people's difficulties in living independently from their parents, and the ways in which their circumstances inhibited their future life ambitions.

The interactivity of online focus groups poses challenges for both moderators and participants, as they defy conventions of conversational turn-taking and are characterised by simultaneous, overlapping responses (or 'threading') (Matt and Stewart, 2000). Where researchers select online methods as a research technique, they must be alive to the ways in which this impacts on the knowledge produced. As such, quick typing skills are essential for the moderator and participants, as well as access to appropriate technology. In our experience, participants seemed able to keep pace with discussions and there were no observable differences in levels of contribution on the basis of demographic traits such as age or gender. However, those who lacked access to a computer or internet connection, or confidence and skills with computers had expressed a preference for a telephone interview. This highlights the need to combine online methods with traditional methods to avoid exclusion. 
p. 24. Online focus groups and qualitative research in the social sciences: their merits and limitations in a study of housing and youth

\section{The use of interactive stimulus}

An additional exercise to facilitate interaction was the use of a virtual whiteboard as a stimulus for discussion. This whiteboard is embedded through the focus groups, with a text-based chat window below, as shown in Figure 1.

Figure 1: The virtual setting for online focus groups

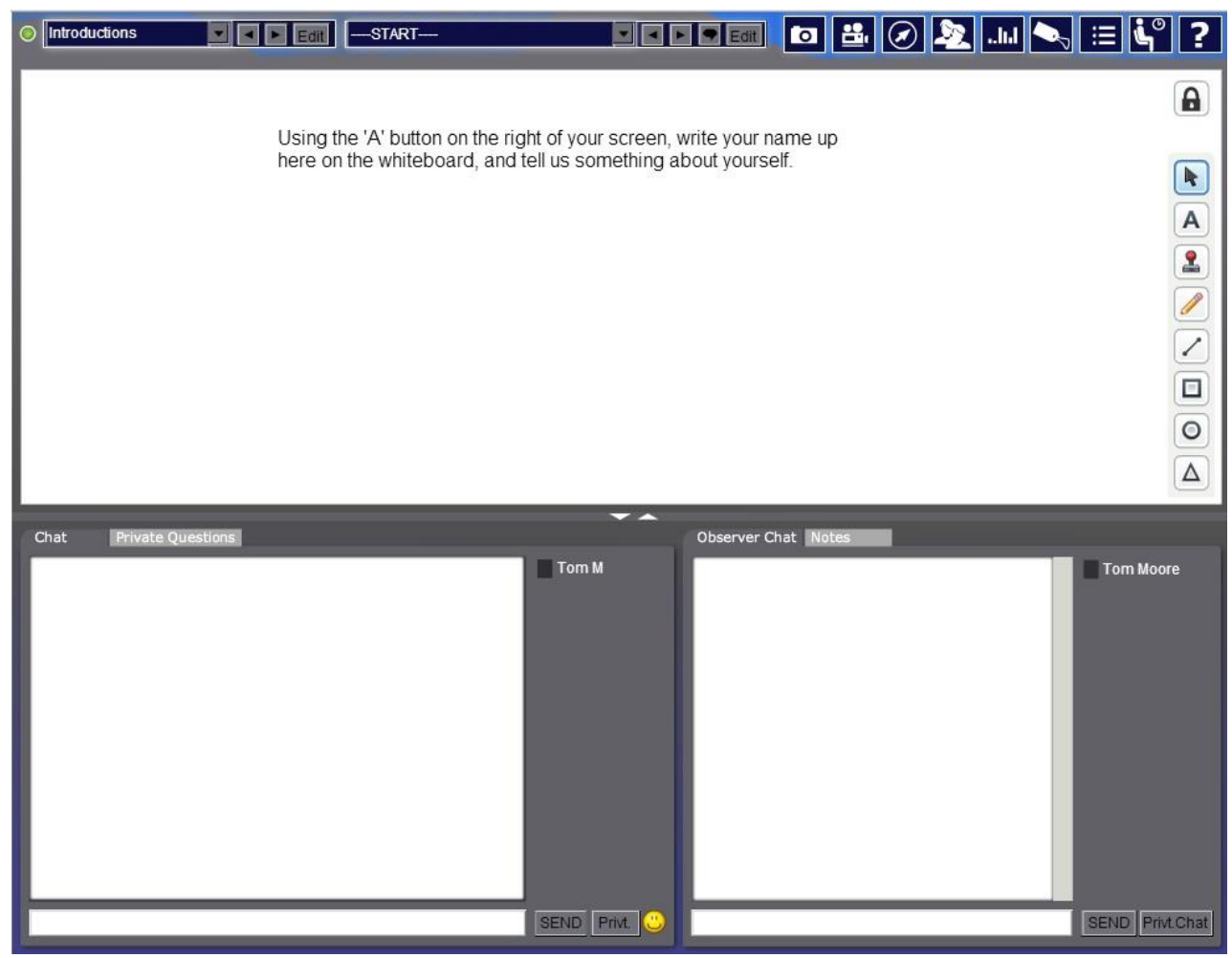

Participants were asked to brainstorm perceptions or thoughts on a whiteboard, which were then discussed in more detail and depth in the chat windows. This is illustrated in Figure 2, where participants were asked to compare differences in housing opportunities and financial welfare for 18-35-year-olds of their generation, compared to their parents' generation. 
p. 25. Online focus groups and qualitative research in the social sciences: their merits and limitations in a study of housing and youth

Figure 2: The use of a virtual whiteboard as an interactive stimulus in online focus groups

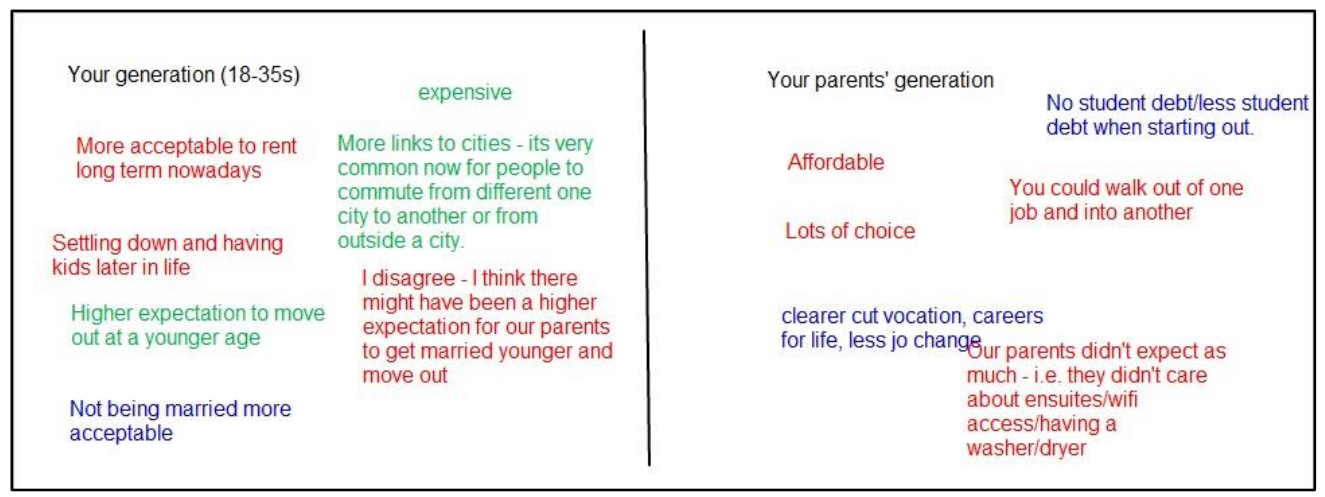

This facilitated group interactions, as participants responded to comments made by each other on the whiteboard, sometimes in agreement but often out of disagreement or lack of clarity. The use of an interactive stimulus encouraged participants to converse with each other, through questioning and defence of particular views, and these interpretations or justifications of material contributed to the richness of our data. It also enabled participants to lead the production of knowledge through visual interaction, as opposed to more formulaic questioning and answering led by the moderator. The whiteboard was also a useful tool for moderation, assisting in introducing or returning to topics of interest, particularly in the context of rapid simultaneous messages and responses, especially in a larger group where its speed and dynamism created several threads of discussion. The whiteboard allowed the moderator to direct the flow of the focus group in a more subtle manner than conventional questioning of participants, therefore supporting the methodological goal of facilitating group interactions to produce data, whilst focusing on the study's key research questions.

However, in some sessions participants did not develop a familiarity with the whiteboard and did not contribute as extensively as others. This led to prolonged periods of relative silence and inactivity. As we were unable to perceive people's body language, there was uncertainty as to the cause (for example, whether silence was due to a lack of engagement with the tool, or whether they were contemplating possible contributions). In other sessions, the use of the whiteboard disrupted conversational coherence and the flow of group interactions, and on reflection there were times when our moderation and introduction of the whiteboard were too interventionist.

This highlights a key limitation for moderators of online focus groups. There is an assumed level of digital competency, which trusts that participants are able to keep track of and manage online and visual interactions. Given that online focus groups emphasise the role of group interactions in the construction of knowledge, there is a need to minimise the amount of direct questioning to prevent the group falling into a directive question and answer session, thereby losing the richness of participant-led interaction. Yet, moderators also need to judge when best to intervene in discussions in order to keep participants on track and ensure planned topics are addressed within the timeframe of the group. Online moderators need to maintain a critical reflexive awareness throughout their focus groups and acknowledge that their judgements and interventions will affect the knowledge that is produced. 
p. 26. Online focus groups and qualitative research in the social sciences: their merits and limitations in a study of housing and youth

\section{Concluding remarks}

Synchronous online focus groups offer a number of advantages for social science researchers. First, they are beneficial in overcoming temporal and spatial barriers to qualitative research. The majority of our groups were geographically distant, and the time and complexity of the data collection would have been greater with conventional face-to-face focus groups.

Second, online methodologies capitalise on increasing societal use of the internet as a powerful medium for communication and group interaction. Through a mix of online and offline recruitment, we were able to engage young people in our study, including those who may be excluded from traditional methods. Our successful use of social networking websites for participant recruitment suggests that further use of social media in qualitative research could be productive for social science researchers. However, online methods are not a panacea for engaging a greater number of participants or for overcoming participation barriers such as participant availability and non-attendance, and face their own issues related to digital exclusion. Our experience highlights that online methodologies do not eliminate the need for offline, nonelectronic forms of qualitative research.

Third, synchronous online focus groups can provide rich exchanges, elucidating the subjective viewpoints of participants. Our creative use of interactive visual stimulus also suggests that they can be valuable platforms for facilitating dynamic group interactions and creating new forms of knowledge. Participants queried and contested each other's statements, offering different interpretations and justifications of each other's viewpoints.

Despite these advantages, text-based interactions make it difficult for researchers to incorporate and work with non-verbal cues in their interactions with participants. Critical reflexivity and acknowledgement of these issues needs to be incorporated into the analysis and presentation of online data, for they affect the ways in which questions are phrased, the development of group dynamics, and the interpretations that are made by researchers.

The lack of human interaction also has implications for the role of moderators. Online scenarios may be useful for encouraging the interactivity of focus groups and for empowering participant-led interactions, but this depends on the individual personalities of those taking part, their proficiency with technology, and the appropriateness of interventions made by moderators seeking to direct focus group discussions. In this sense, as with challenges in sampling and recruitment, online settings may still encounter similar barriers to conventional qualitative research.

This paper has offered a reflexive account of the use of online focus groups in a study of young people's housing access and financial welfare opportunities. While this method has been popularised in areas such as health research and psychology, it has not been utilised to any great extent in other areas of the social sciences such as human geography, sociology, social policy, and urban studies. This paper has advanced our understanding of the advantages, limitations and implications of online methodologies, and their appropriateness for research with different populations, highlighting a range of considerations that should be incorporated into their future use in qualitative research. 
p. 27. Online focus groups and qualitative research in the social sciences: their merits and limitations in a study of housing and youth

* Correspondence Addresses:

Dr Tom Moore, University of Sheffield, ICOSS, 219 Portobello, Sheffield, S1 4DP. Email: tom.moore@sheffield.ac.uk

Dr Kim McKee, University of St Andrews, The Observatory, Centre for Housing Research, St Andrews, Buchanan Gardens, KY16 9LZ. Email: km410@st-andrews.ac.uk

Dr Pauline McLoughlin, RMIT University, School of Globan, Urban and Social Studies, GPO Box 2476, Melbourne Victoria 3001, Australia. Email: pj.mcloughlin@gmail.com

\section{References}

Bagnoli, A. and Clark, A. (2010) Focus groups with young people: a participatory approach to research planning. Journal of Youth Studies, 13, 1, 101-19.

Bedford, T. and Burgess, J. (2001) The focus group experience. In: M. Limb and C. Dwyer (eds) Qualitative methodologies for geographers: issues and debates. New York: Arnold: 121-35.

Bosco, F. and Herman, T. (2010) Focus groups as collaborative research performances. In: D. DeLyser (ed) The Sage Handbook of Qualitative Geography. London: Sage, 193-08.

Bourdieu, P. (1992) An Invitation to Reflexive Sociology. Cambridge: Polity Press.

The British Psychological Society Ethics Guidelines for Internet-mediated Research. Available at: http://www.bps.org.uk/system/files/Public\%20files/inf206guidelines-for-internet-mediated-research.pdf [Accessed: 16/04/15].

Carey, M. (1995) Comment: Concerns in the Analysis of Focus Group Data. Qualitative Health Research, 5, 4, 487-95.

Fern, E. (2001) Advanced Focus Group Research. London: Sage.

Fox, F., Morris, M. and Rumsey, N. (2007) Doing synchronous online focus groups with young people: methodological reflection. Qualitative Health Research, 17, 4, 53947.

Gaiser, T. (1997) Conducting online focus groups: a methodological discussion. Social Science Computer Review, 15, 2, 135-44.

Goss, J. and Leinbach, T. (1996) Focus groups as alternative research practice: experience with transmigrants in Indonesia. Area, 28, 2, 115-23.

Holbrook, B. and Jackson, P. (1996) Shopping around: focus group research in North London. Area, 28, 2, 136-42.

Hollander, J. (2004) The social context of focus groups. Journal of Contemporary Ethnography, 33, 602-37.

Hopkins, P. (2007) Thinking critically and creatively about focus group. Area, 39, 4, 528-35.

Kamberelis, G. and Dimitriadis, G. (2013) Focus Groups: From structured interviews to collective conversations. New York: Routledge.

Kenny, A. (2005) Interaction in cyberspace: an online focus group. Journal of Advanced Nursing, 49, 4, 414-22.

Koch, N. (2013) Technologising the opinion: focus groups, performance and free speech. Area, 45, 4, 411-18.

McKee, K. (2012) Young People, Homeownership and Future Welfare. Housing Studies, $27,6,853-62$.

Mann, C. and Stewart, F. (2000) Internet Communication and Qualitative Research: A Handbook for Researching Online. London: Sage.

Moore, T. (2013) Where are young people living? Understanding the housing options of 18-35-year olds in the UK. Wealth Gap Briefing Series. Available at: 
p. 28. Online focus groups and qualitative research in the social sciences: their merits and limitations in a study of housing and youth

http://wealthgap.wp.st-

andrews.ac.uk/files/2013/02/WealthGap No 05 Where are young people livi ng.pdf. [Accessed: 04/10/14]

Morgan, D. (1996) Focus Groups. Annual Review of Sociology, 22, 129-52.

O'Connor, A., Jackson, L., Goldsmith, L. and Skirton, H. (2014) Can I get a retweet please? Health research recruitment and the Twittersphere. Journal of Advanced Nursing, 70, 3, 599-09.

Office for National Statistics (2013) Internet Access - Households and Individuals, 2012 part 2. Available at: http://www.ons.gov.uk/ons/dcp171778 301822.pdf [Accessed: 04/10/14].

Townsend, L., Sathiaseelan, A., Fairhurst, G. and Wallace, C. (2013) Enhanced broadband access as a solution to the social and economic problems of the rural digital divide. Local Economy, 28, 6, 580-95.

Williams, S., Clausen, M., Robertson, A., Peacock, S. and McPherson, S. (2012) Methodological reflections on the use of asynchronous online focus groups in health research. International Journal of Qualitative Methods, 11, 4, 369-83. 